\title{
ESTIMATIVA DA PARTIÇÃO DE ENERGIA NA SUPERFÍCIE A \\ PARTIR DE SÉRIES METEOROLÓGICAS DE TEMPERATURA E
}

\section{UMIDADE ESPECÍFICA DO AR}

\author{
Daiane de Vargas Brondani ${ }^{1}$, Otávio Costa Acevedo ${ }^{1}$ \\ 1Pós-Graduação em Meteorologia, Centro de Ciência Naturais e Exatas \\ Universidade Federal de Santa Maria
}

\section{RESUMO}

Neste trabalho, é proposto um método simples de estimativa da razão entre os fluxos turbulentos de calor sensível (H) e latente (LE) e da altura da camada limite planetária (CLP), utilizando séries meteorológicas de temperatura ( $\mathrm{T}$ ) e umidade específica do ar (q). De forma geral, o método desenvolvido reproduziu satisfatoriamente a evolução diária de $\mathrm{T}$ e q, o que faz deste método promissor para estimativas da partição de energia em superfície, bem como da altura da CLP. No entanto, é necessária a validação do método a partir de medidas de $\mathrm{H}$ e LE, assim como alguns ajustes devem ser feitos.

\section{SUMMARY}

In this work, it is proposed a simple method for estimation of the ratio between the turbulent fluxes of sensible (H) and latent heat (LE) and the height of the planetary boundary layer (CLP) using weather series of temperature ( $\mathrm{T}$ ) and specific humidity (q). The proposed method reproduced satisfactorily the daily evolution of $\mathrm{T}$ and $\mathrm{q}$, which does this a promising method for estimating the surface energy partition as well as the height of the CLP. However, it is necessary to validate the method from measurements of $\mathrm{H}$ and LE, as well as some adjustments.

\section{INTRODUÇÃO}

As variáveis umidade específica (q) e temperatura do ar (T) estão associadas aos dois principais fluxos de energia na superfície: fluxo de calor latente (LE) e fluxo de calor sensível $(\mathrm{H})$, respectivamente. Atualmente, as técnicas utilizadas para a determinação de $\mathrm{H} \mathrm{e}$ LE além do custo elevado, requerem grande demanda de recursos humanos na sua operação.

Neste trabalho, é proposto um método simples para estimativas da partição dos fluxos superficiais de energia e da altura da camada limite planetária (CLP) utilizando apenas séries meteorológicas de $\mathrm{T}$ e q.

\section{MATERIAL E MÉTODOS}

Os dados horários diários de T e q são provenientes de estações meteorológicas do Instituto Nacional de Meteorologia (INMET) e de radiossondagens lançadas na Base Aérea de Santa Maria (BASM) do período setembro de 2007 a agosto de 2009. Neste último, 
conjunto de dados interpolação linear foi determinada entre a altura de 100 a 2500 metros para as variáveis de $\mathrm{T}$ e q.

O método utilizado aqui tem por base a hipótese de que as evoluções temporais médias de $\mathrm{T}$ e q são controladas unicamente pela convergência dos fluxos superficiais de $\mathrm{He}$ LE, respectivamente, visto que é razoável supor que na média para um dado mês, na região em estudo, os termos advectivos se cancelam. E, desta forma, os ciclos diários de T e q são controlados somente pelos fluxos superficiais e sua convergência na CLP: $\partial \bar{\theta} / \partial t=-\partial\left(\bar{w}^{\prime} \theta^{\prime}\right)_{0} / \partial z$ e $\partial \overline{\mathbf{q}} / \partial \mathrm{t}=-\partial\left(\overline{\mathbf{w}^{\prime} \mathbf{q}^{\prime}}\right)_{0} / \partial z$, em que ${ }^{\partial\left(\overline{\mathbf{w}^{\prime} \theta^{\prime}}\right)_{0}} \quad \mathrm{e}^{\partial\left(\overline{\mathbf{w}^{\prime} \mathbf{q}^{\prime}}\right)_{0}}$ são respectivamente os fluxos superficiais de H e LE. A integração das equações acima na vertical, entre a superfície e o topo da CLP, $h$, leva a $\partial\left(\overline{\mathbf{w}^{\prime} \theta^{\prime}}\right)_{0}=\mathrm{h} \partial \hat{\theta} / \partial \mathrm{t}$ e $\partial\left(\overline{\left.\mathbf{w}^{\prime} \mathbf{q}^{\prime}\right)_{0}}=\mathrm{h} \partial \overline{\mathrm{q}} / \partial \mathrm{t}\right.$. Como $h$ é desconhecido, utilizaram-se aproximações para sua variação ao longo do dia, como proporcional à raiz quadrada do tempo (Tennekes, 1973).

Assim, foi possível testar diversas evoluções temporais simuladas por um modelo simples, nos quais foram variadas a altura máxima da CLP ao final do dia e a razão de Bowen (B), que relaciona os fluxos superficiais de H e LE. Os valores dessas variáveis que permitiam um melhor ajuste do modelo aos ciclos diários observados de $\mathrm{T}$ e $\mathrm{q}$ foram considerados como nossas estimativas para essas variáveis.
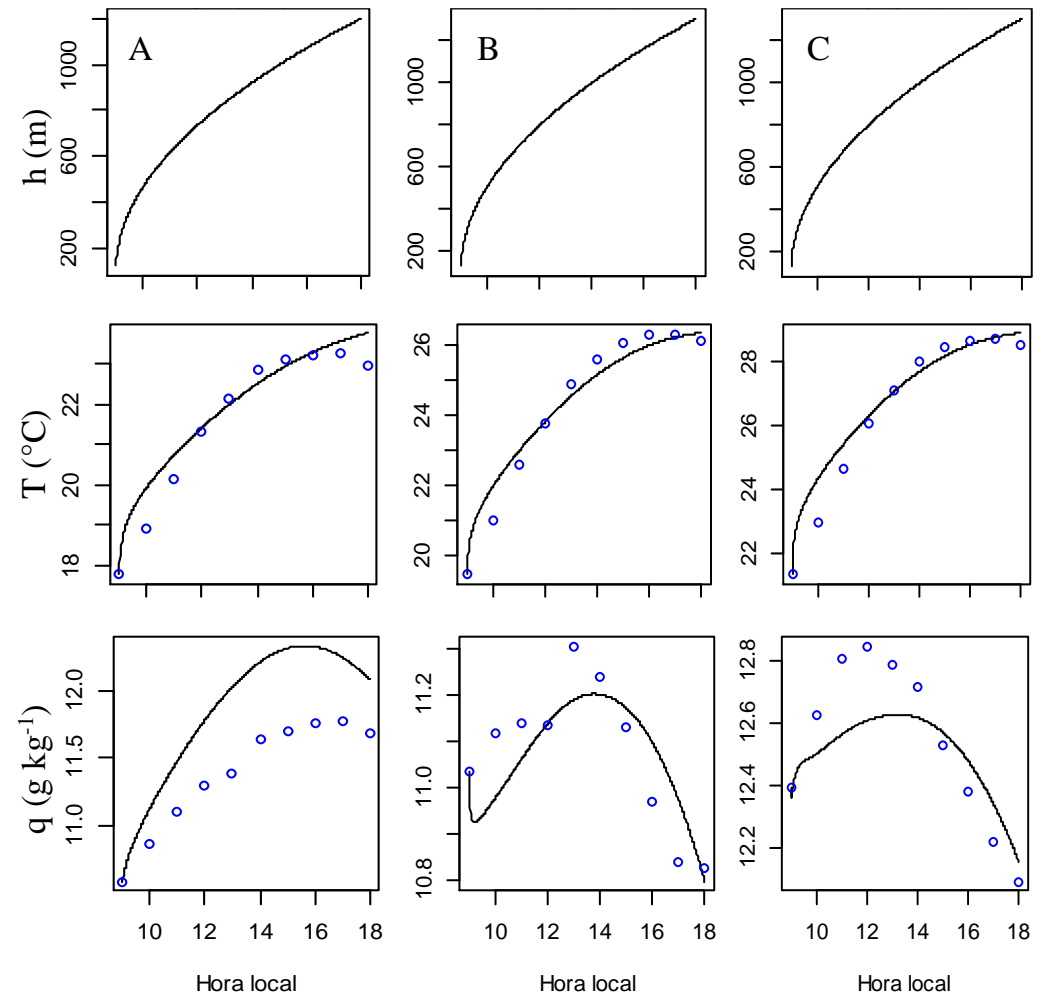

Figura 1. Evolução horária da simulação (curvas) de $h\left(1^{\mathrm{a}}\right.$ linha), de $\mathrm{T}\left(\mathrm{T}, 2^{\mathrm{a}}\right.$ linha) e q (q, $3^{\mathrm{a}}$ linha) para os meses de outubro a dezembro (Colunas A, B e C) a partir de dados de radiossondagem e estação de superfície (pontos em azul) em Santa Maria-RS. As curvas foram obtidas considerando-se a razão de Bowen de 0,2, 0,6 e 0,5 e $h$ igual a 1200, 1300 e $1300 \mathrm{~m}$, respectivamente para os meses de outubro a dezembro. Estes valores foram escolhidos por serem os melhores ajustes encontrados para o menor valor da raiz quadrada do erro quadrático médio. 


\section{RESULTADOS}

Os resultados das simulações que melhor se ajustaram à evolução das séries meteorológicas podem ser vistos na Figura 1 em que as colunas A, B, e C correspondem respectivamente a outubro, novembro e dezembro. Nota-se que o método proporcionou estimativa da altura da CLP, bem como de uma estimativa adequada da razão de Bowen que resultou em um bom ajuste entre os valores estimados e observados de $\mathrm{T}$ e q.

\section{CONSIDERAÇÕES FINAIS}

De forma geral, o método desenvolvido reproduziu satisfatoriamente a evolução diária de T e q, o que o torna promissor para estimativa da partição de energia na superfície, bem como da altura da CLP. No entanto, é necessária a validação do método a partir de medidas de $\mathrm{H}$ e LE, assim como demais ajustes.

\section{AGRADECIMENTOS}

À Fundação de Amparo à Pesquisa do Estado do Rio Grande do Sul (FAPERGS) pela concessão da bolsa de pós-graduação.

\section{REFERÊNCIAS BIBLIOGRÁFICAS}

TENNEKES, H., A model for the dynamics of the inversion above a convective boundary layer. Journal of the atmospheric sciences, v.30, p.558-567, 1973. 